\title{
SOME EXACT SOLUTIONS DESCRIBING UNSTEADY PLANE GAS FLOWS WITH SHOCKS*
}

\author{
BY
}

\author{
P. L. SACHDEV AND A. VENKATASWAMY REDDY \\ Indian Institute of Science, Bangalore
}

\begin{abstract}
Summary. A new class of exact solutions of plane gasdynamic equations is found which describes piston-driven shocks into non-uniform media. The governing equations of these flows are taken in the coordinate system used earlier by Ustinov, and their similarity form is determined by the method of infinitesimal transformations. The solutions give shocks with velocities which either decay or grown in a finite or infinite time depending on the density distribution in the ambient medium, although their strength remains constant. The results of the present study are related to earlier investigations describing the propagation of shocks of constant strength into non-uniform media.
\end{abstract}

1. Introduction. One-dimensional unsteady anisentropic gas flows have been studied for a long time. Some of the solutions describing these flows may be found in Sedov [1], Keller [2], McVittie [3] for plane, cylindrical and spherical geometries. It would appear, therefore, that new exact solutions, in the similarity form or otherwise, are difficult to find, at least in the physical plane. The hodograph transformation would render the boundary condtions, at the piston driving the flows, difficult to satisfy. Thus, one has to look for such a transformed system as would allow the boundary conditions, both at the piston and at the shock, to be conveniently satisfied. Such a transformed system was given by Ustinov [4]. The choice of independent variables in the new system was such that the shock trajectory could be obtained by keeping one of the coordinates, $s$, constant, while the piston path (and the particle trajectories) were marked out by constant values of $\tau$, the other coordinate. Ustinov also found a special class of solutions which describe gas flows into a non-uniform undisturbed medium, produced by a piston and headed by a shock. Some flows without shocks were also found by allowing the shock strength to tend to zero.

In a different class, Castell and Rogers [5] have constructed some new solutions for one-dimensional flows from the known solutions of Sedov, using certain reciprocal and adjoint transformations and following an earlier study of Ustinov [6].

In the present paper, we generalize the work of Ustinov [4]. We first find all the similarity transformations for the equations of Ustinov by applying the method of infinitesimal transformations (Bluman and Cole [7]). We find that only two types of similarity variables made up of $\tau$ and $s$ are compatible with shock conditions, the so-called power law type and the exponential type. The similarity forms of these solutions can be easily guessed

* Received September 17, 1981. This work was carried out in part under the University Grants Commission Project on 'Wave Phenomena' No. 23-884/78 CSRIF-Code No. 001/Maths/78. 
by direct intuitive argument, but the method of infinitesimal transformations ensures that there are no other nontrivially different similarity forms. We construct similarity solutions in these variables consistent with the shock conditions. The power law solutions include Ustinov's solution as a special case and in general involve five arbitrary constants. The exponential solutions involve four arbitrary constants. These (constant) parameters are exclusive of $\gamma$, the ratio of specific heats. The two classes of solutions describe piston-driven shocks into media which are at rest and have constant pressure but variable distributions of density. In a medium with increasing density the shock velocities decay, while they grow in a medium with decreasing density. However, in either case, the shock strength measured by the pressure ratio across the shock or the Mach number remains constant along the shock trajectory. In this sense, the family of solutions considered here describes the propagation of shocks of constant strength which are produced by a suitable piston motion such that the inhomogeneity of the medium ahead and the motion of the piston behind contrive to maintain such shocks. We also consider solutions without shocks in the limit of vanishing shock strength, and local analysis is carried out to describe approximately the nature of these solutions. The physical nature of the solutions described in the present paper is brought out in a number of graphs showing particle trajectories and isobars for a variety of representative parameters.

In Sec. 2, we give the basic equations and boundary conditons and their transformation to new variables. Sec. 3 gives briefly the theory of infinitesimal transformations, as applied to Ustinov's equations, to identify the similarity variables. Sec. 4 deals with power law solutions while Sec. 5 treats the exponential solutions. Finally, Sec. 6 contains discussion and conclusions of the present study.

2. Basic equations and their transformation. The equations governing plane, compressible and inviscid flows are

$$
\begin{gathered}
\rho_{t}+(\rho u)_{x}=0, \\
\rho\left(u_{t}+u u_{x}\right)+p_{x}=0 . \\
S_{t}+u S_{x}=0, \\
p=\rho^{\gamma} \exp \left(\frac{S-S_{0}}{C_{v}}\right) .
\end{gathered}
$$

Here $\rho, u, p$ and $S$ are the density of the fluid, particle velocity, pressure and entropy respectively at any point $x$ and time $t$. Eq. (2.4) relates the thermodynamic variables $p, \rho$ and $S ; \gamma$ and $C_{v}$ stand for the ratio of specific heats and the specific heat at constant volume respectively. In view of (2.1) and a certain combination of (2.1) and (2.2), we introduce the variables $\tau$ and $\xi$ :

$$
\begin{aligned}
& d \tau=\rho d x-(\rho u) d t, \\
& d \xi=\rho u d x-\left(p+\rho u^{2}\right) d t .
\end{aligned}
$$

These differential relations imply

$$
\begin{aligned}
& d x=-\frac{u}{p} d \xi+\frac{\left(p+\rho u^{2}\right)}{\rho p} d \tau, \\
& d t=-\frac{d \xi}{p}+\frac{u}{p} d \tau .
\end{aligned}
$$


The Rankine-Hugoniot conditions holding across a shock moving into a non-uniform medium with density $\rho_{*}=\rho_{*}(x), p=p_{0}$ (a constant) and $u_{0}=0$ are

$$
\begin{gathered}
\frac{\rho}{\rho_{*}}=\frac{(\gamma+1) p+(\gamma-1) p_{0}}{(\gamma-1) p+(\gamma+1) p_{0}} \\
u=\left(\frac{2}{\rho_{*}}\right)^{1 / 2}\left(p-p_{0}\right)\left[(\gamma+1) p+(\gamma-1) p_{0}\right]^{-1 / 2}, \\
U=\left(2 \rho_{*}\right)^{-1 / 2}\left[(\gamma+1) p+(\gamma-1) p_{0}\right]^{1 / 2} .
\end{gathered}
$$

Writing the shock trajectory $d x=U d t$ in terms of the variables $\xi$ and $\tau$ via (2.7)-(2.8), we have

$$
d \xi+\phi(\tau) d \tau=0
$$

where

$$
\phi(\tau)=\frac{p_{0}}{\rho_{*}(x)} U^{-1} .
$$

To facilitate the fitting of the shock conditions, a new coordinate $s$ is introduced such that

$$
d s=d \xi+\phi(\tau) d \tau,
$$

implying $s$ is constant along the shock. The differential relations (2.7)-(2.8) now become

$$
\begin{aligned}
d x & =-\frac{u}{p} d s+\frac{\left(p+\rho u^{2}+\rho u \phi\right)}{\rho p} d \tau, \\
d t & =-\frac{d s}{p}+\frac{u+\phi}{p} d \tau .
\end{aligned}
$$

We remark that Eq. (2.14) is invariant under a translation in $s$ so that we may, for convenience, choose $s=0$ to give the shock trajectory. Eq. (2.5) implies that $\tau=$ constant along a particle line. Thus, the trajectories $\tau=$ constant also give lines with constant entropy in the $\tau-s$ plane. In consequence of this, Eq. (2.3) may be dropped and the other two equations (2.1)-(2.2) may be transformed as

$$
\begin{gathered}
w_{\tau}+w w_{s}-\frac{f}{\gamma} p^{-1 / \gamma} p_{s}-\phi^{\prime}(\tau)=0, \\
p_{\tau}+w p_{s}-p w_{s}=0
\end{gathered}
$$

where

$$
f(\tau)=\left[p \rho^{-\gamma}\right]^{1 / \gamma}, \quad w=u+\phi
$$

from the equation of state (2.4). Here $\phi(\tau)$ is as defined in (2.13). Thus, we have to deal with the second-order system $(2.17)-(2.18)$ wherein the functions $\phi(\tau)$ and $f(\tau)$, arbitrary so far, will be determined from the shock conditions. Thus, $(2.9)-(2.11)$ lead to

$$
\begin{gathered}
p_{s=0}=\frac{p_{0}}{\gamma+1}\left(\frac{2 p_{0}}{\rho_{*} \phi^{2}}-\gamma+1\right), \\
w_{s=0}=\phi\left(\frac{p_{s=0}}{p_{0}}\right),
\end{gathered}
$$




$$
f(\tau)=p_{s=0}^{1 / \gamma}\left[\frac{(\gamma-1) p_{0}+2 \gamma \rho_{*} \phi^{2}}{(\gamma+1) p_{0} \rho_{*}}\right] .
$$

Since we seek similarity solutions in terms of a combination of variables $\tau$ and $s$, we choose $\phi(\tau), f(\tau)$ and $\rho_{*}(\tau)$ at the shock such that $\rho_{*} \phi^{2}$ and $f / \phi^{2}$ are constant.

3. Invariance group and similarity variables. To identify the similarity form, we use the theory of infinitesimal transformations given originally by Ovsjannikov [8] and expounded more clearly by Bluman and Cole [7]. We employ, in fact, an abbreviated form of the same, as in the paper of Logan and Perez [9]. We seek a one-parameter infinitesimal group of transformations

$$
\bar{\tau}=\tau+\varepsilon T, \quad \bar{s}=s+\varepsilon S ; \quad \bar{w}=w+\varepsilon W, \quad \bar{p}=p+\varepsilon P,
$$

where the generators $T, S, W$, and $P$ are functions of $\tau, s, u$ and $p$ under which the system of equations (2.17) $-(2.18)$ are invariant. We shall further impose the requirements that the shock trajectory is given by the similarity variable equal to zero (cf. Sec. 2) and the (reduced) dependent variables assume constant values along the shock. As usual, the similarity transformations will reduce the PDEs (2.17)-(2.18) to ODEs which may be solved analytically, if possible, or numerically otherwise.

We introduce the following notation for the sake of convenience. Let $x^{1}=\tau, x^{2}=s$, $u^{1}=w, u^{2}=p$ and define the derivatives

$$
p_{j}^{i}=\partial u^{i} / \partial x^{j}, \quad i=1,2, \quad j=1,2 .
$$

It can be verified that the invariance of the differential equations, as computed by Bluman and Cole, is equivalent to the following definition of invariance [9]: a system of differential equations

$$
H_{n}\left(x^{i}, u^{j}, p_{j}^{i}\right)=0 \quad n=1,2,
$$

is said to be constantly conformally invariant under the infinitesimal group (3.1) if there exist constants $\alpha_{n j}(n, j=1,2)$ such that

$$
L H_{n}=\alpha_{n j} H_{j}, \quad n=1,2,
$$

for all smooth surfaces $u^{i}=u^{i}\left(x^{j}\right)$, where $L$ is the Lie derivative in the direction of the extended vector field,

$$
L=A=\xi_{x}^{i} \frac{\partial}{\partial x^{i}}+\xi_{u}^{j} \frac{\partial}{\partial u^{j}}+\xi_{p j}^{i} \frac{\partial}{\partial p_{j}^{i}}
$$

where

$$
\xi_{x}^{1}=T, \quad \xi_{x}^{2}=S, \quad \xi_{u}^{1}=W, \quad \xi_{u}^{2}=P
$$

and where

$$
\xi_{p j}^{i}=\frac{\partial \xi_{u}^{i}}{\partial x^{j}}+\frac{\partial \xi_{u}^{i}}{\partial u^{k}} p_{j}^{k}-\frac{\partial \xi_{x}^{k}}{\partial x^{j}} p_{k}^{i}-\frac{\partial \xi_{x}^{k}}{\partial u^{m}} p_{k}^{i} p_{j}^{m}
$$

are the generators of the derivative transformations. Writing out (3.2) gives

$$
\frac{\partial H_{n}}{\partial x^{j}} \xi_{x}^{j}+\frac{\partial H_{n}}{\partial u^{i}} \xi_{u}^{i}+\frac{\partial H_{n}}{\partial p_{j}^{i}} \xi_{p j}^{i}=\alpha_{n j} H_{j}, \quad n=1,2 .
$$


If $\xi_{p j}^{i}$ from Eq. (3.3) are substituted into (3.4), polynomials in $p_{j}^{i}$ are obtained. If these equations are made to hold for arbitrary values of $p_{j}^{i}$, thus requiring their coefficients to vanish, a system of linear PDEs in the generators $T, W, P$ and $S$ are obtained. This system of equations, often referred to as the determining system, is solved to find the group (3.1).

If the above analysis is carried out for Eq. (2.17), then the invariance of this equation gives

$$
\begin{gathered}
T=T(\tau, s), \quad S=S(\tau, s), \\
\frac{\partial W}{\partial \tau}+w \frac{\partial W}{\partial s}-\frac{f}{\gamma} p^{-1 / \gamma} \frac{\partial P}{\partial s}=-\alpha_{11} \phi^{\prime}+T \phi^{\prime \prime}, \\
\frac{\partial W}{\partial w}-\frac{\partial T}{\partial t}-w \frac{\partial T}{\partial s}=\alpha_{11}, \\
\frac{\partial W}{\partial p}+\frac{f}{\gamma} p^{-1 / \gamma} \frac{\partial T}{\partial s}=\alpha_{12}, \\
-\frac{\partial S}{\partial \tau}+w \frac{\partial W}{\partial w}-w \frac{\partial S}{\partial s}-\frac{f}{\gamma} p^{-1 / \gamma} \frac{\partial P}{\partial w}=\alpha_{11} w-\alpha_{12} p-W, \\
w \frac{f W}{\partial p}-\frac{f}{\gamma} p^{-1 / \gamma} \frac{\partial P}{\partial p}+\frac{f}{\gamma} p^{-1 / \gamma} \frac{\partial S}{\partial s} \\
=T \frac{f^{\prime}}{\gamma} p^{-1 / \gamma}-\frac{f}{\gamma^{2}} p^{-(\gamma+1) / \gamma} P-\alpha_{11} \frac{f}{\gamma} p^{-1 / \gamma}+\alpha_{12} w .
\end{gathered}
$$

In the same way, invariance of Eq. (2.18) yields

$$
\begin{gathered}
\frac{\partial P}{\partial \tau}+w \frac{\partial P}{\partial s}-p \frac{\partial W}{\partial s}=-\alpha_{21} \phi^{\prime} \\
\frac{\partial P}{\partial w}+p \frac{\partial T}{\partial s}=\alpha_{21} \\
\frac{\partial P}{\partial p}-\frac{\partial T}{\partial \tau}-w \frac{\partial T}{\partial s}=\alpha_{22} \\
w \frac{\partial P}{\partial p}-p \frac{\partial W}{\partial w}+p \frac{\partial S}{\partial s}=\alpha_{21} w+\left(1-\alpha_{22}\right) p \\
-\frac{\partial S}{\partial \tau}+w \frac{\partial P}{\partial p}-w \frac{\partial S}{\partial s}-p \frac{\partial W}{\partial p}=-\alpha_{21} \frac{f}{\gamma} p^{-1 / \gamma}+\alpha_{22} w-W .
\end{gathered}
$$

We now solve the above determining differential equations for the transformation group. Eqs. (3.12) and (3.13) give $\partial T / \partial s=0$. This result with (3.5) gives $T=T(\tau)$. Eqs. (3.14) and (3.7) imply $\alpha_{21}=0$, which in turn gives $\partial P / \partial w=0$. Eq. (3.9) and (3.8) give $\partial W / \partial p=$ $-\alpha_{12}=\alpha_{12}=0$. Again, (3.7) and (3.11) with $\partial P / \partial w=0$ imply $\partial P / \partial s=0$. Then, (3.6) and (3.11) give $W=W(\tau, w), P=P(p)$. It is also clear from the determining equations (3.5)(3.15) that

$$
\frac{\partial^{2} P}{\partial p^{2}}=\frac{\partial^{2} W}{\partial w^{2}}=\frac{\partial^{2} T}{\partial \tau^{2}}=\frac{\partial^{2} S}{\partial s^{2}}=0
$$


Hence

$$
T=b_{1} \tau+b_{2} .
$$

Eq. (3.16), with the help of (3.12) and (3.10), implies

$$
P=\left(\alpha_{22}+b_{1}\right) p .
$$

Eqs. (3.14) and (3.9) imply $\partial S / \partial s=\left(2 b_{1}+\alpha_{11}\right)$, that is

$$
S=\left(2 b_{1}+\alpha_{11}\right) s+S_{1}(\tau)
$$

Now, (3.9) gives

$$
W=\left(\alpha_{11}+b_{1}\right) w+S^{\prime}(\tau)
$$

Finally, (3.10) and (3.6) give

$$
\begin{gathered}
f(\tau)=a_{1} T^{\left.\left(\left(\alpha_{22}+b_{1}\right) / \gamma\right)+2 \alpha_{11}+b_{1}-\alpha_{22}\right) / b_{1}}, b_{1} \neq 0, \\
\phi(\tau)=\int T^{\alpha_{11} / b_{1}}\left[\int \frac{S_{1}^{\prime \prime}(\tau) d \tau}{T^{\alpha_{11} / b_{1}+1}}+a_{2}\right] d \tau+a_{3} \\
f(\tau)=a_{1} \exp \left[\left(\frac{\alpha_{22}+b_{1}}{\gamma}+2 \alpha_{11}+b_{1}-\alpha_{22}\right) \frac{\tau}{b_{2}}\right] \quad, b_{1}=0 . \\
\phi(\tau)=\int \exp \left(\frac{\alpha_{11} \tau}{b_{2}}\right)\left[\int \exp \left(\frac{-\alpha_{11} \tau}{b_{2}}\right) \frac{S_{1}^{\prime \prime}(\tau)}{b_{2}} d \tau+a_{2}\right] d \tau+a_{3}
\end{gathered}
$$

In the above, $a_{1}, a_{2}, a_{3}, b_{1}$ and $b_{2}$ are arbitrary constants and $S_{1}(\tau)$ is an arbitrary function of $\tau$. Eqs. (3.16) $-(3.19)$ give the generators of the invariance group for the differential equations (2.17) and (2.18). The invariant surface conditions [7] to determine the similarity variable and the similarity solution for $w$ and $p$ become, respectively,

$$
\begin{gathered}
T \frac{\partial w}{\partial \tau}+S \frac{\partial w}{\partial s}=W \\
T \frac{\partial p}{\partial \tau}+S \frac{\partial p}{\partial s}=P
\end{gathered}
$$

The characteristic system of (3.22) is

$$
\frac{d \tau}{b_{1} \tau+b_{2}}=\frac{d s}{\left(\alpha_{11}+2 b_{1}\right) s+S_{1}(\tau)}=\frac{d W}{\left(\alpha_{11}+b_{1}\right) w+S_{1}^{\prime}(\tau)} .
$$

One first integral gives the similarity variable

$$
\begin{aligned}
\sigma & =s T^{-\alpha_{11} / b_{1}-2}-\int S_{1}(\tau) T^{-\alpha_{11} / b_{1}-3}, \quad b_{1} \neq 0 \\
& =s \exp \left(-\frac{\alpha_{11}}{b_{2}} \tau\right)-\int \exp \left(-\frac{\alpha_{11}}{b_{2}} \tau\right) \frac{S_{1}(\tau)}{b_{2}} d \tau, \quad b_{1}=0
\end{aligned}
$$


The second first integral gives

$$
\begin{aligned}
w(\tau, s) & =T^{\alpha_{11} / b_{1}+1}\left[\int T^{\alpha_{11} / b_{1}+2} S_{1}(\tau) d \tau+W_{1}(\sigma)\right], \quad b_{1} \neq 0, \\
& =\exp \left(\frac{\alpha_{22} \tau}{b_{2}}\right)\left[\int \exp \left(\frac{\alpha_{11} \tau}{b_{2}}\right) \frac{S_{1}^{\prime}(\tau)}{b_{2}} d \tau+W_{1}(\sigma)\right], \quad b_{1}=0 .
\end{aligned}
$$

In a similar way Eq. (3.23) gives

$$
\begin{aligned}
p(\tau, s) & =T^{\alpha_{22} / b_{1}+1}+P_{1}(\sigma), & & b_{1} \neq 0, \\
& =\exp \left(\frac{\alpha_{22}}{b_{2}} \tau\right) P_{1}(\sigma), & & b_{1}=0 .
\end{aligned}
$$

In the above, $W_{1}$ and $P_{1}$ are arbitrary functions of $\sigma$. Next we use the shock conditions $(2.20)-(2.22)$ to determine $S_{1}(\tau)$ and other constants. That the similarity variable $\sigma$ is constant on the trajectory $s=0$ implies

$$
\begin{gathered}
S_{1}(\tau)=0, \\
\sigma=s T^{-\left(\alpha_{11} / b_{1}+2\right)}, \quad b_{1} \neq 0, \\
=s \exp \left(-\alpha_{11} / b_{2}\right) \tau, \quad b_{1}=0 .
\end{gathered}
$$

Also, from the shock conditions we have

$$
f / \phi^{2}=\text { constant }
$$

which requires that $\alpha_{22}+b_{1}=0$ and $a_{3}=0$. Without loss of generality, we take $b_{2}=1$ and $\tau=0$ at the origin.

Finally, the similarity forms of the solutions for the differential equations $(2.17)-(2.18)$ are given by

$$
\begin{aligned}
\sigma & =s\left[1+b_{1} \tau\right], \\
w & =\left[1+b_{1} \tau\right]^{\alpha_{11 / b}+2} W_{1}(\sigma), \quad b_{1} \neq 0 \\
p & =P_{1}(\sigma), \\
f & =a_{1}\left[1+b_{1} \tau\right]^{2 \alpha_{11} / b_{1}+2}, \\
\phi & =a_{2}\left[1+b_{1} \tau\right]^{\alpha_{11} / b_{1}+1} . \\
\sigma & =s \exp \left(-\alpha_{11} \tau\right), \\
w & =\exp \left(\alpha_{11} \tau\right) W_{1}(\sigma), \\
p & =P_{1}(\sigma), \\
f & =a_{1} \exp \left(2 \alpha_{11} \tau\right), \\
\phi & =a_{2} \exp \left(\alpha_{11} \tau\right) .
\end{aligned}
$$


4. Power-law similarity solutions. Introducing the similarity form of the solutions

$$
\begin{aligned}
\sigma & =\frac{s}{p_{0} t_{0}}\left(1+\frac{a \tau}{\rho_{0} x_{0}}\right)^{-(1+\alpha)}, \\
w & =\phi W(\sigma), \\
p & =p_{0} P(\sigma), \\
\phi & =\frac{p_{0} t_{0}}{\rho_{0} x_{0}}\left(1+\frac{a \tau}{\rho_{0} x_{0}}\right)^{\alpha}, \\
f & =\frac{\gamma p_{0}^{1 / \gamma}}{b \rho_{0}} C_{1}\left(1+\frac{a \tau}{\rho_{0} x_{0}}\right)^{2 \alpha},
\end{aligned}
$$

in accordance with (3.27), into (2.17) and (2.18), we obtain

$$
\begin{gathered}
(W-a(\alpha+1) \sigma) W^{\prime}-C_{1} P^{-1 / \gamma} P^{\prime}=a \alpha(1-W), \\
P W^{\prime}-(W-(\alpha+1) a \sigma) P^{\prime}=0 .
\end{gathered}
$$

$W$ and $P$ here should not be confused with those of Sec. 3. Here they refer to the similarity form of $w$ and $p$ as defined in (4.2) and (4.3). In the above, $\alpha$ is the exponent in the definition of the similarity variable and $x_{0}, t_{0}, p_{0}$ and $\rho_{0}$ are arbitrary constants with the dimensions of distance, time, pressure and density respectively. The constant $a$ assumes values +1 or -1 . The parameter $C_{1}$ is given by

$$
C_{1}=P_{*}^{1 / \gamma}\left(\frac{\gamma-1+(2 \gamma / b)}{\gamma+1}\right) \frac{b}{\gamma},
$$

where the pressure ratio across the shock

$$
\frac{p_{s=0}}{p_{0}}=P_{*}=\frac{2 b-\gamma+1}{\gamma+1},
$$

and $b=\rho_{0} x_{0}^{2} / p_{0} t_{0}^{2}$.

The undisturbed distribution of density $\rho_{*}(\tau)$ is chosen to fit into the similarity form of the shock conditions. Thus,

$$
\rho_{*}=\rho_{0}\left(1+\frac{a \tau}{\rho_{0} x_{0}}\right)^{-2 \alpha} .
$$

The family of solutions referred to thus involves five arbitrary constants, namely $x_{0}, t_{0}, p_{0}$, $\rho_{0}$ and $\alpha \neq 0$. The case $\alpha=0$ would imply isentropy of the flow, which is not admissible by the basic transformations of Sec. 2 .

The initial conditions for the ordinary differential equations (4.6) and (4.7) can be found by the substitution of the similarity form of the solution (4.1)-(4.5) and (4.8) into the shock conditions, (2.20)-(2.22). The shock trajectory is taken to be $s=0$, implying $\sigma=0$, and along it

$$
W(0)=P(0)=\frac{2 b-\gamma+1}{\gamma+1}=P_{*} .
$$

Taking $\sigma, W$ as dependent variables and $P$ as independent variable, (4.6), (4.7) and (4.9) can 
be written as

$$
\begin{gathered}
d W / d P=(W-V) / P, \\
d V / d P=a_{1}\left(C_{1} P^{(\gamma-1) / \gamma}-(W-V)^{2}\right) / P(W-1),
\end{gathered}
$$

with initial conditions

$$
V(P)=0, \quad W\left(P_{*}\right)=P_{*},
$$

where $V=(\alpha+1) a \sigma$ and $a_{1}=(\alpha+1) / \alpha$. Along the shocks $s=0$, therefore, from (2.5) we have

$$
d \tau=\rho_{*} d x
$$

Substituting $\rho_{*}$ from (4.8) into (4.13) and integrating, we get

$$
\begin{aligned}
\frac{x}{x_{0}} & =\frac{1}{a(2 \alpha+1)}\left[\left(1+\frac{a \tau}{\rho_{0} x_{0}}\right)^{2 \alpha+1}-1\right], \quad \alpha \neq-\frac{1}{2}, \\
& =1 / a \ln \left(1+a \tau / \rho_{0} x_{0}\right), \quad \alpha=-\frac{1}{2} .
\end{aligned}
$$

Again, using $d x=U d t$ in (4.13), we get, after an integration,

$$
\begin{aligned}
\frac{t}{t_{0}} & =\frac{1}{a(\alpha+1)}\left[\left(1+\frac{a \tau}{\rho_{0} x_{0}}\right)^{\alpha+1}-1\right], & & \alpha \neq-1, \\
& =\frac{1}{a} \ln \left(1+\frac{a \tau}{\rho_{0} x_{0}}\right), & \alpha & =-1 .
\end{aligned}
$$

In the derivation of Eqs. (4.14) and (4.15), the boundary conditions $\tau=0$ at $x=0, t=0$ have been used. Eqs. (4.14)-(4.15) give the parametric representation of the shock wave motion. Eliminating $\tau$ between Eqs. (4.14) and (4.15), we get

$$
\begin{aligned}
\frac{x(t)}{x_{0}} & =\frac{1}{a(2 \alpha+1)}\left\{\left[1+a(\alpha+1) \frac{t}{t_{0}}\right]^{(2 \alpha+1) /(\alpha+1)}-1\right\}, & & \alpha \neq-1,-\frac{1}{2}, \\
& =\frac{1}{a}\left(1-\exp \left(-a t / t_{0}\right)\right), & & \alpha=-1, \\
& =\frac{2}{a} \ln \left(1+\frac{a t}{t_{0}}\right), & & \alpha=-\frac{1}{2},
\end{aligned}
$$

as the explicit trajectory of the shock.

Expressing $\tau$ as a function of $x$ from Eqs. (4.14) and substituting into (4.8), we get

$$
\begin{aligned}
\rho_{*}(x) & =\rho_{0}\left[1+a(2 \alpha+1) \frac{x}{x_{0}}\right]^{-(2 \alpha / 2 \alpha+1)}, & & \alpha \neq-\frac{1}{2}, \\
& =\rho_{0} \exp \left(\frac{a x}{x_{0}}\right), & \alpha & =-\frac{1}{2} .
\end{aligned}
$$

Similarly, expressing $\tau$ as a function of $t$ from Eq. (4.15) and substituting into the expression 
for shock velocity, we get

$$
\begin{aligned}
U & =\frac{x_{0}}{t_{0}}\left[1+a(\alpha+1) \frac{t}{t_{0}}\right]^{\alpha / \alpha+1}, & & \alpha \neq-1, \\
& =\frac{x_{0}}{t_{0}} \exp \left(-\frac{a t}{t_{0}}\right), & & \alpha=1 .
\end{aligned}
$$

It can be verified that, when $a \alpha>0$, the undisturbed density $\rho_{*}$ ahead of the wave is a decreasing function of $x$, the shock velocity $U$ increases with $t$, and the non-dimensionalized pressure, at the back of the shock, $P=p / p_{0}>P_{*}$. Similarly, when $a \alpha<0, \rho_{*}$ increases with $x, U$ decreases with $t$ and $1<P<P_{*}$ at the back of the shock. Figs. 1-7 give the distribution of pressure and particle trajectories between the shock locus (or sonic line) and the piston path for some typical values of the parameters.

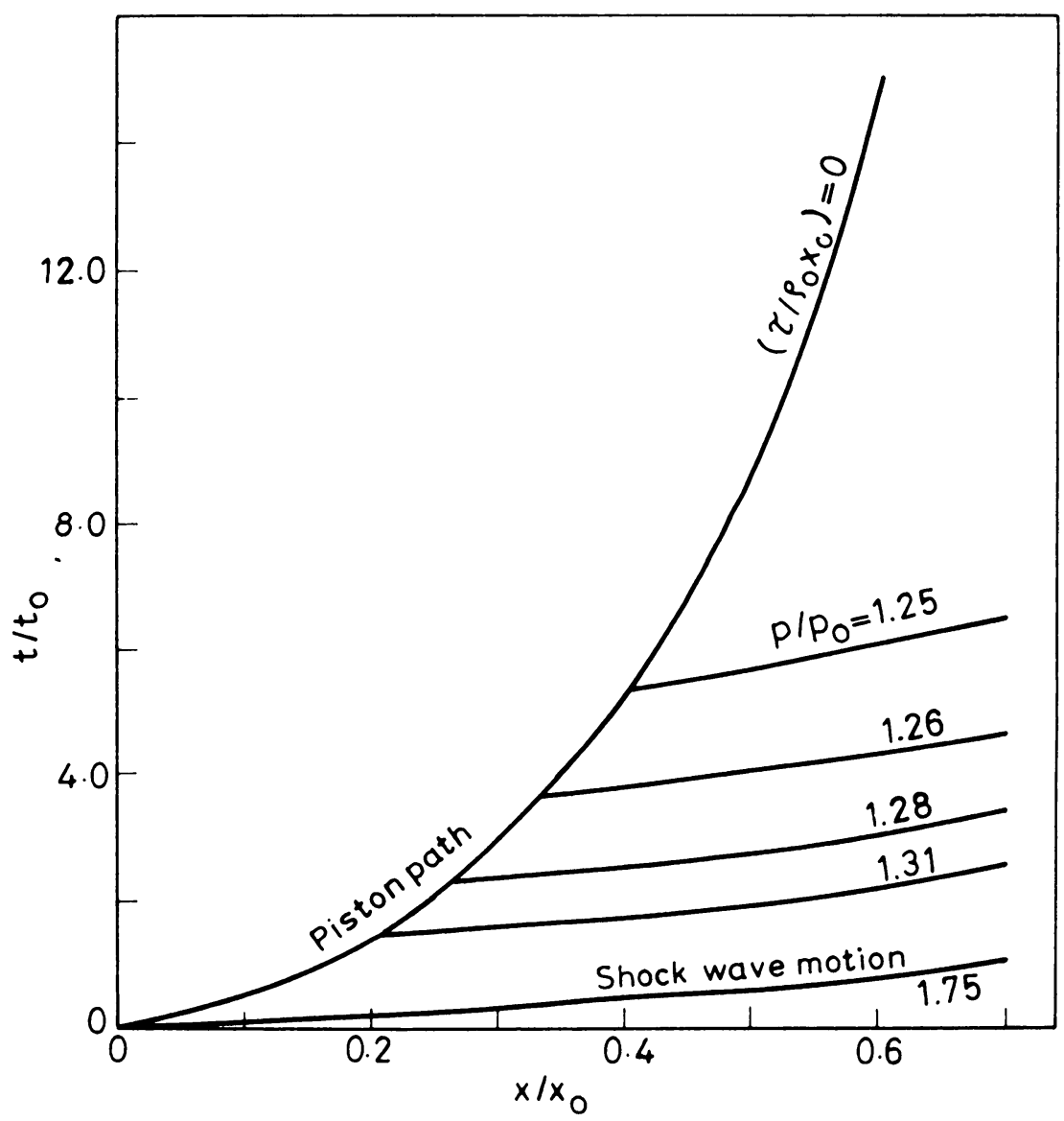

Fig. 1. Piston path, isobars and shock locus for $\gamma=\frac{5}{3}, b=\frac{8}{3}, \alpha=-\frac{1}{2}, a=1$ (Eqs. (4.26)). The shock velocity decays to zero in an infinite time and distance in an increasing density medium. The density becomes infinite at an infinite distance. 


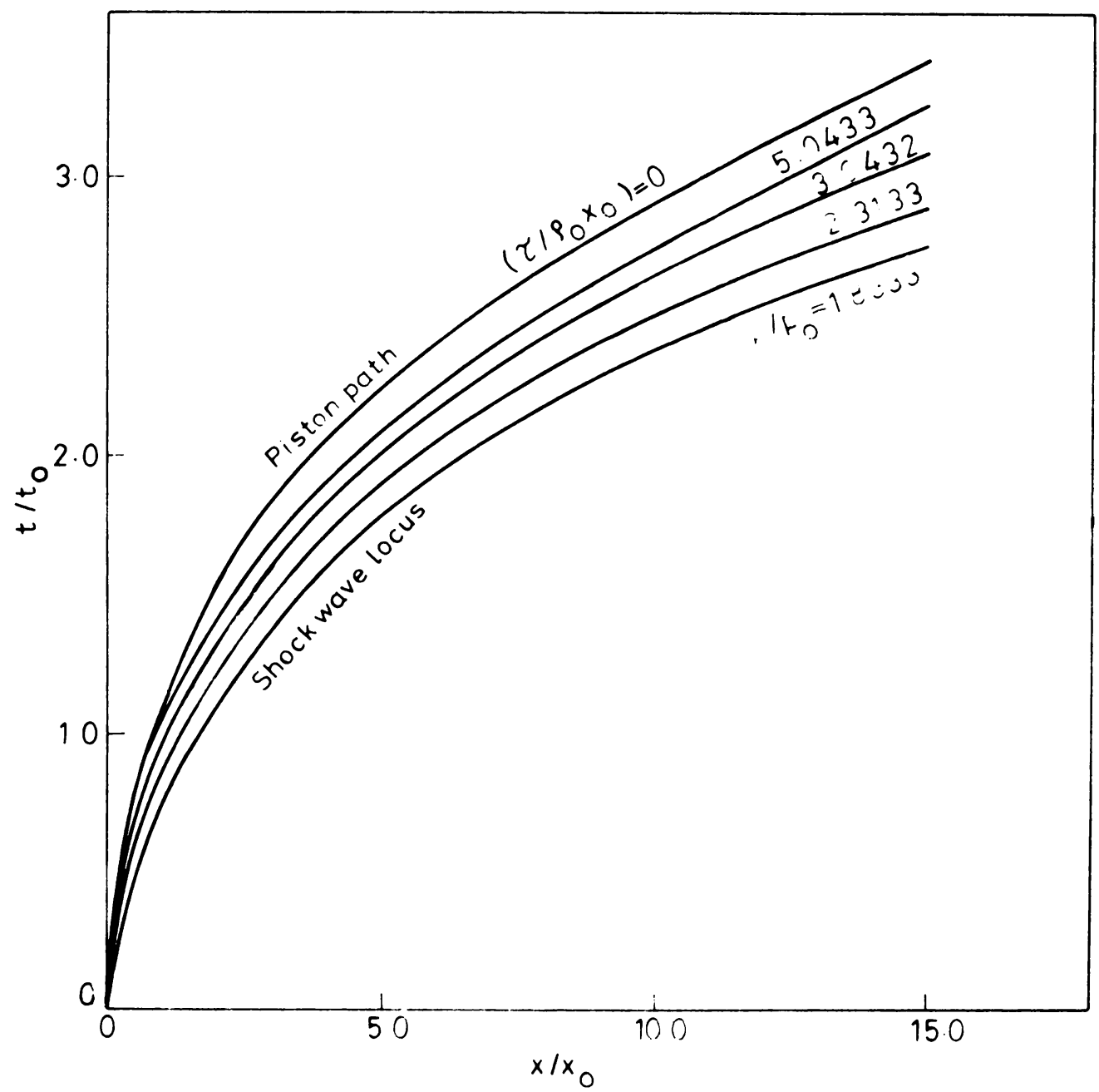

FIG. 2. As in Fig. 1 with $\gamma=1.4, b=2.4, \alpha=-1, a=-1$ (Eqs. 4.25)). The shock velocity grows to infinity in an infinite time and distance in a decreasing density medium. The density becomes zero at an infinite distance.

We substitute

$$
s=p_{0} t_{0} \sigma\left(1+\frac{a \tau}{\rho_{0} x_{0}}\right)^{\alpha+1}, \quad u=\phi(W-1), \quad p=p_{0} P
$$

into Eqs. (2.15) and (2.16) to get

$$
\begin{aligned}
& \frac{\partial x}{\partial P}=-\frac{x_{0}}{b}\left(\frac{W-1}{P}\right)\left(1+\frac{a \tau}{\rho_{0} x_{0}}\right)^{2 \alpha+1} \frac{d \sigma}{d P}, \\
& \frac{\partial t}{\partial P}=-\frac{t_{0}}{P}\left(1+\frac{a \tau}{\rho_{0} x_{0}}\right)^{\alpha+1} \frac{d \sigma}{d P} .
\end{aligned}
$$




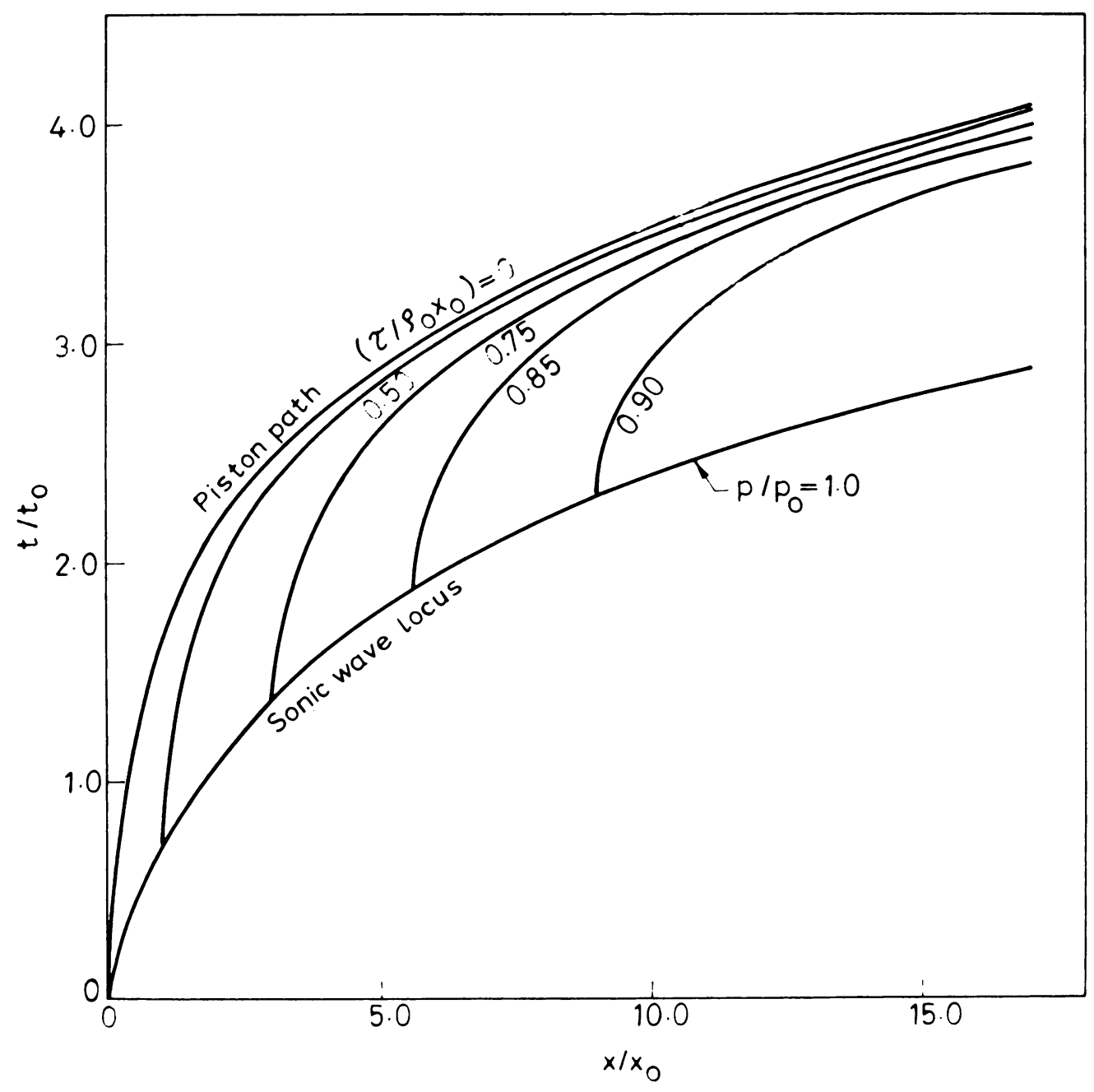

Fig. 3. Piston path, particle trajectories and sonic wave locus for $\gamma=b=1.4, \alpha=-1, a=-1$. The sonic speed grows to infinity in an infinite time and distance in a decreasing density medium.

Using Eqs. (4.10) and (4.11), (4.19) can be written as

$$
\begin{aligned}
& \frac{\partial x}{\partial P}=\frac{x_{0}}{a \alpha b}\left[\left(\frac{d W}{d P}\right)^{2}-C_{1} P^{-(\gamma+1 / \gamma)}\right]\left[1+\frac{\alpha \tau}{\rho_{0} x_{0}}\right]^{2 \alpha+1}, \\
& \frac{\partial t}{\partial P}=\frac{t_{0}}{a \alpha}\left[\left(\frac{d W}{d P}\right)^{2}-C_{1} P^{-(\gamma+1 / \gamma)}\right]\left[1+\frac{\alpha \tau}{\rho_{0} x_{0}}\right]^{\alpha+1} /(W-1) .
\end{aligned}
$$

Making use of the conditions (4.14) and (4.15) at the shock $P=P_{*}$, Eqs. (4.20) and (4.21) 
can be integrated to give

$$
\begin{aligned}
\frac{x}{x_{0}}= & \frac{\left(1+\frac{a \tau}{\rho_{0} x_{0}}\right)^{2 \alpha+1}}{a b \alpha} \int_{P_{*}}^{P}\left[\left(\frac{d W}{d P}\right)^{2}-C_{1} P^{-(\gamma+1 / \gamma)}\right] d P & \\
& +\frac{1}{a(2 \alpha+1)}\left[\left(1+\frac{a \tau}{\rho_{0} x_{0}}\right)^{2 \alpha+1}-1\right], & \alpha \neq-\frac{1}{2}, \\
= & -\frac{2}{a b} \int_{P_{*}}^{P}\left[\left(\frac{d W}{d P}\right)^{2}-C_{1} P^{-(\gamma+1 / \gamma)}\right] d P+\frac{1}{a} \ln \left(1+\frac{a \tau}{\rho_{0} x_{0}}\right), & \alpha=-\frac{1}{2},
\end{aligned}
$$

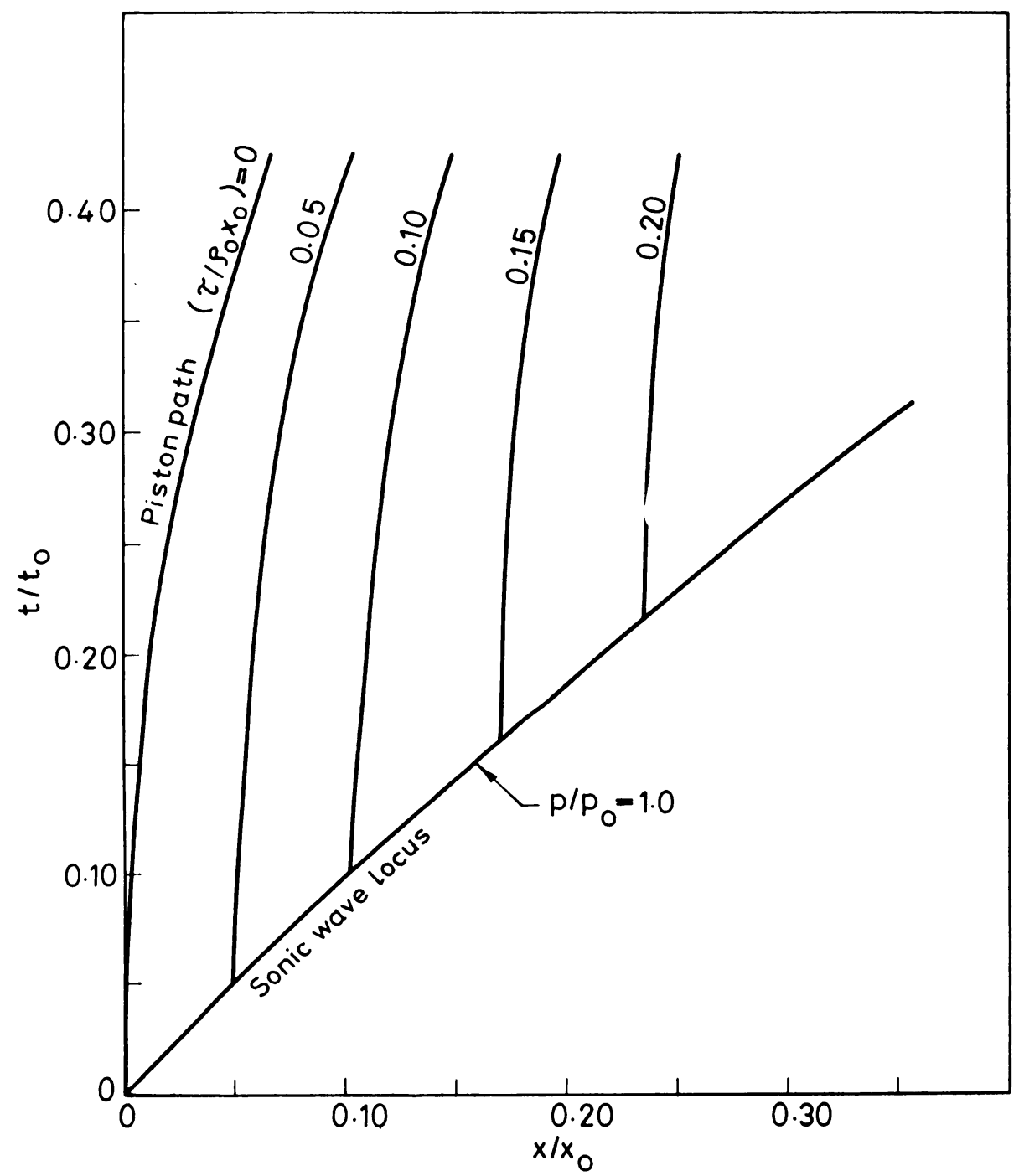

Fig. 4. As in Fig. 3 with $\gamma=b=1.4, \alpha=-0.75, a=-1$ (Eqs. (4.16), (4.22) and (4.23)). The sonic speed grows to infinity in a finite time $\left(t=4 t_{0}\right)$ and infinite distance in a decreasing density medium. The density becomes infinite at an infinite distance. 


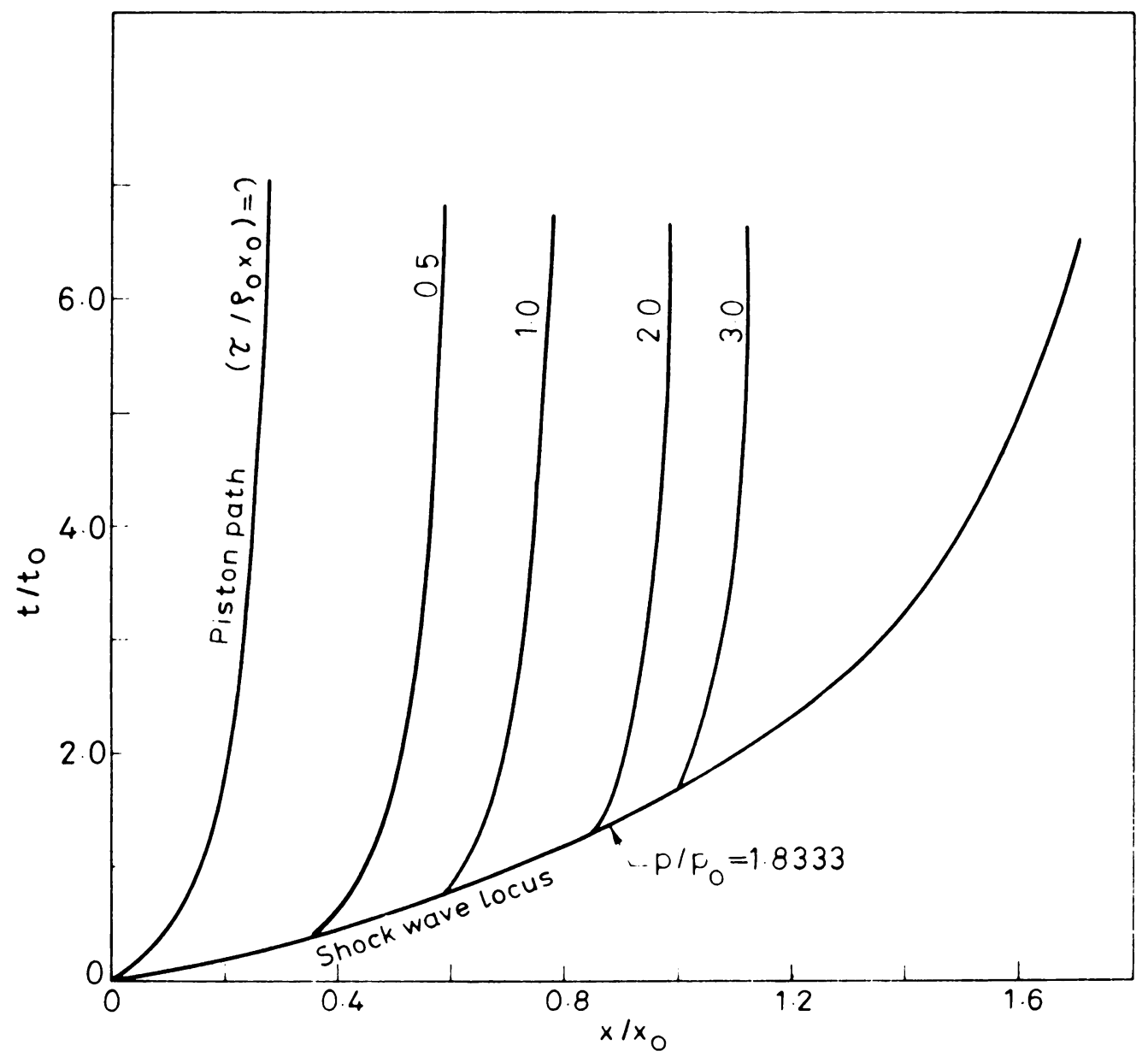

Fig. 5. Piston path, particle trajectories and shock locus for $\gamma=1.4, b=2.4, \alpha=-0.75, a=1$. The shock velocity decays to zero in an infinite time and finite distance $\left(x=2 x_{0}\right)$ in an increasing density medium. The density becomes infinite at $x=2 x_{0}$.

$$
\begin{array}{rlr}
\frac{t}{t_{0}}= & \frac{\left(1+\frac{a \tau}{\rho_{0} x_{0}}\right)^{\alpha+1}}{a \alpha} \int_{P_{*}}^{P}\left[\left(\frac{d W}{d P}\right)^{2}-C_{1} P^{-(\gamma+1 / \gamma)}\right] \frac{d P}{(W-1)} & \\
& +\frac{1}{a(\alpha+1)}\left[\left(1+\frac{a \tau}{\rho_{0} x_{0}}\right)^{\alpha+1}-1\right], & \alpha \neq-1, \\
= & -\frac{1}{a} \int_{P *}^{P}\left[\left(\frac{d W}{d P}\right)^{2}-C_{1} P^{-(\gamma+1 / \gamma)}\right] \frac{d P}{(W-1)}+\frac{1}{a} \ln \left(1+\frac{a \tau}{\rho_{0} x_{0}}\right), & \alpha=-1
\end{array}
$$

where

$$
\begin{array}{cll}
P_{*} \leq P<\infty & \text { for } & a \alpha>0, \\
1<P \leq P_{*} & \text { for } & a \alpha<0 .
\end{array}
$$


Setting $\tau=$ constant in (4.22) and (4.23), we obtain the trajectories of the gas particles in parametric form. In particular, with $\tau=0$, we obtain the law of motion of the piston as

$$
\begin{aligned}
& \frac{x(P)}{x_{0}}=\frac{1}{a b \alpha} \int_{P_{*}}^{P}\left[\left(\frac{d W}{d P}\right)^{2}-C_{1} P^{-(\gamma+1 / \gamma)}\right] d P, \\
& \frac{t(P)}{t_{0}}=\frac{1}{a \alpha} \int_{P_{*}}^{P}\left[\left(\frac{d W}{d P}\right)^{2}-C_{1} P^{-(\gamma+1 / \gamma)}\right] \frac{d P}{(W-1)},
\end{aligned}
$$

which gives rise to a shock wave moving according to (4.16).

Thus, Eqs. (4.4), (4.5), (4.8), (4.10)-(4.11) and (4.22)-(4.23) give in a closed form the exact solution of the following problem in terms of some quadratures: at $t=0$, there is a quiescent gas in the region $x \geq 0$ with constant pressure equal to $p_{0}$ and density determined by the relation (4.17). At the time $t=0$, a piston begins to move according to the law (4.24) in the region $x>0$. Ahead of the piston a shock appears immediately whose law of motion is given by Eq. (4.16). The solution of the problem contains five arbitrary constants $x_{0}, t_{0}, p_{0}$, $\rho_{0}$ and $\alpha$.

If $M_{0}$ denotes the ratio of the shock velocity to the sound velocity in the quiescent gas ahead of it, we find that $M_{0}=(b / \gamma)^{1 / 2}$. In view of the second law of thermodynamics $M_{0}>1$, that is, $b>\gamma$.

Eqs. (4.10) and (4.11) can be solved explicitly for $\alpha=-1$ and $\alpha=-\frac{1}{2}$. For $\alpha=-1$, the solution is given by

$$
\begin{aligned}
\frac{x}{x_{0}} & =\frac{\left(1+\frac{a \tau}{\rho_{0} x_{0}}\right)^{-1}}{a b}\left[\gamma C_{1}\left(P_{*}^{-1 / \gamma}-P^{-1 / \gamma}\right)-\left(P-P_{*}\right)\right]+\frac{1}{a}\left[1-\left(1+\frac{a \tau}{\rho_{0} x_{0}}\right)^{-1}\right] \\
\frac{t}{t_{0}} & =\frac{1}{a} \int_{P_{*}}^{P}\left[C_{1} P^{-(\gamma+1 / \gamma)}-1\right] \frac{d P}{(P-1)}+\frac{1}{a} \ln \left(1+\frac{a \tau}{\rho_{0} x_{0}}\right) \\
u & =\phi(P-1), \quad \phi=\frac{p_{0} t_{0}}{\rho_{0} x_{0}}\left(1+\frac{a \tau}{\rho_{0} x_{0}}\right)^{-1}, \\
f(\tau)= & \frac{\gamma p_{0}^{1 / \gamma}}{b \rho_{0}} C_{1}\left(1+\frac{a \tau}{\rho_{0} x_{0}}\right)^{-2}, \quad \rho_{*}=\rho_{0}\left(1-\frac{a x}{x_{0}}\right)^{-2}, \\
\frac{x(t)}{x_{0}}= & \frac{1}{a}\left[1-\exp \left(-\frac{a t}{t_{0}}\right)\right], \quad \text { and } \quad P_{*} \leq P<\infty \quad \text { for } a=-1 .
\end{aligned}
$$

We recover Ustinov's solution [4] by taking $a=-1$ in (4.25).

For $\alpha=-\frac{1}{2}$, the solution is again explicit:

$$
\begin{aligned}
\frac{x}{x_{0}} & =\frac{2}{a b} \int_{P_{*}}^{P}\left[C_{1} P^{-(\gamma+1) / \gamma}-\frac{\left(b-\gamma C_{1} P^{-1 / \gamma}\right)^{2}}{Q}\right] d P+\frac{1}{a} \ln \left(1+\frac{a \tau}{\rho_{0} x_{0}}\right), \\
\frac{t}{t_{0}} & =\frac{2\left(1+\frac{a \tau}{\rho_{0} x_{0}}\right)^{1 / 2}}{a} \int_{P_{*}}^{P}\left[C_{1} P^{-(\gamma+1 / \gamma)}-\frac{\left(b-\gamma C_{1} P^{-1 / \gamma}\right)^{2}}{Q}\right] \frac{d P}{Q^{1 / 2}}
\end{aligned}
$$




$$
\begin{aligned}
&+\frac{2}{a}\left[\left(1+\frac{a \tau}{\rho_{0} x_{0}}\right)^{1 / 2}-1\right], \\
& u= \phi Q(P)^{1 / 2}, \quad \phi=\frac{p_{0} t_{0}}{\rho_{0} x_{0}}\left(1+\frac{a \tau}{\rho_{0} x_{0}}\right)^{-1 / 2}, f=\frac{\gamma}{b}-\frac{p_{0}^{1 / \gamma}}{\rho_{0}} C_{1}\left(1+\frac{a \tau}{\rho_{0} x_{0}}\right)^{-1}, \\
& \rho_{*}=\rho_{0} \exp \left(\frac{a x}{x_{0}}\right), \quad \frac{X(t)}{x_{0}}=\frac{2}{a} \ln \left(1+\frac{a t}{t_{0}}\right), \\
& 1<P \leq P_{*}, \text { for } a=1, \text { and } P_{*} \leq P<\infty \text { for } a=-1,
\end{aligned}
$$

where

$$
Q=1+\left(\frac{\gamma+1}{\gamma-1}\right) P_{*}+2 b P-\frac{2 \gamma^{2} C_{1} P^{(\gamma-1) / \gamma}}{\gamma-1} .
$$

We now consider the limiting case when the shock strength tends to zero so that the front is not a shock but a characteristic. In this case $M_{0}=1$, that is $b=\gamma$, and the shock degenerates into a sonic wave. Eq. (4.11) has a singularity at $P=P_{*} \equiv 1$, and therefore we cannot directly solve (4.10) and (4.11) numerically. We find the local solution of (4.10) and (4.11) in the neighbourhood of $P=1$, when $W=1, V=0$ and utilize it to start the solution numerically.

It is easy to verify that the local solution of (4.10) and (4.11) for the shockless case is given by

$$
\begin{aligned}
W & =1+(P-1)+b_{2}(P-1)^{2}+b_{3}(P-1)^{3}+O(P-1)^{4}, \\
V & =-2 b_{2}(P-1)-\left(b_{2}+3 b_{3}\right)(P-1)^{2}-\left(2 b_{3}+4 b_{4}\right)(P-1)^{3}+O(P-1)^{4},
\end{aligned}
$$

where

$$
\begin{aligned}
& b_{2}=-\left(\frac{\gamma+1}{\gamma}\right)\left(\frac{a_{1}}{1-2 a_{1}}\right), \quad b_{3}=-\left(\frac{\gamma+1}{12 \gamma}\right) \frac{a_{1}}{\left(1-a_{1}\right)}\left[1+\frac{\gamma+1}{\gamma}\left(\frac{a_{1}-1}{2 a_{1}-1}\right)\right], \\
& b_{4}=\frac{\left(3 a_{1}-2\right)}{4\left(3-2 a_{1}\right)} b_{2} b_{3}+(\gamma+1)(2 \gamma+1)(3 \gamma+1) a_{1} / 24 \gamma^{3}\left(3-2 a_{1}\right) .
\end{aligned}
$$

Making use of (4.27), Eqs. (4.24) can be integrated to give the approximate piston path:

$$
\begin{gathered}
\frac{x(P)}{x_{0}}=\frac{1}{a \alpha} \frac{1}{\gamma a_{1}}\left[b_{2}(P-1)^{2}+\left(2 b_{3}+\frac{2}{3} b_{2}^{2}\right)(P-1)^{3}\right]+O(P-1)^{4}, \\
\frac{t(P)}{t_{0}}=\frac{1}{a \alpha} \frac{1}{a_{1}}\left[2 b_{2}(P-1)+3 b_{3}(P-1)^{2}+4 b_{4}(P-1)^{3}\right]+O(P-1)^{4}, \\
P \geq 1, \quad a \alpha>0 .
\end{gathered}
$$

In (4.28), $x$ and $t$ are positive in the neighborhood of $P=1$ only if $-2<\alpha<0$.

The local solution of (4.10) and (4.11) in the neighborhood of $P=P_{*}>1$ is given by

$$
\begin{aligned}
W & =P_{*}+\left(P-P_{*}\right)+a_{2}\left(P-P_{*}\right)^{2}+a_{3}\left(P-P_{*}\right)^{3}+O\left(P-P_{*}\right)^{4}, \\
V & =-2 a_{2} P_{*}\left(P-P_{*}\right)-\left(a_{2}+3 a_{3} P_{*}\right)\left(P-P_{*}\right)^{2}-\left(2 a_{3}+4 a_{4} P_{*}\right)\left(P-P_{*}\right)^{3}+O\left(P-P_{*}\right)^{4},
\end{aligned}
$$




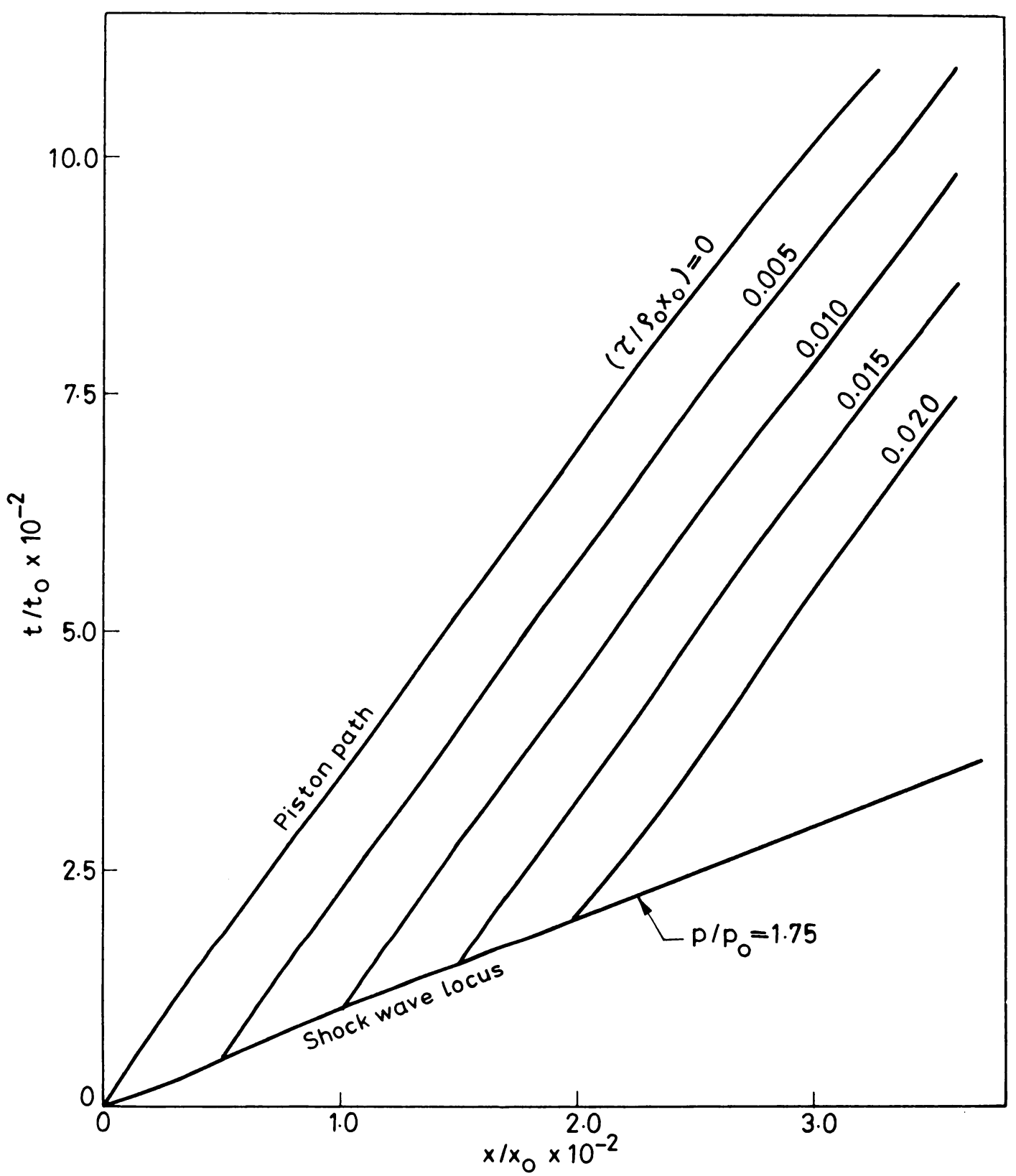

Fig. 6. As in Fig. 5 with $\gamma=\frac{5}{3}, b=\frac{8}{3}, \alpha=-0.25, a=-1$. The shock velocity grows to infinity in a finite time $\left(t=4 t_{0} / 3\right)$ and a finite distance $\left(x=2 x_{0}\right)$ in a decreasing density medium. The density becomes zero at $x=2 x_{0}$.

where

$$
\begin{aligned}
& a_{2}=a_{1}\left(1-C_{1}\right) / 2\left(P_{*}-1\right), \\
& a_{3}=\frac{1}{6\left(P_{*}-1\right)}\left[\frac{a_{1}\left(1-C_{1}\right)}{\left(P_{*}-1\right)}(2 a-1)+\frac{\gamma+1}{\gamma} \frac{a_{1} C_{1}}{P_{*}}\right],
\end{aligned}
$$




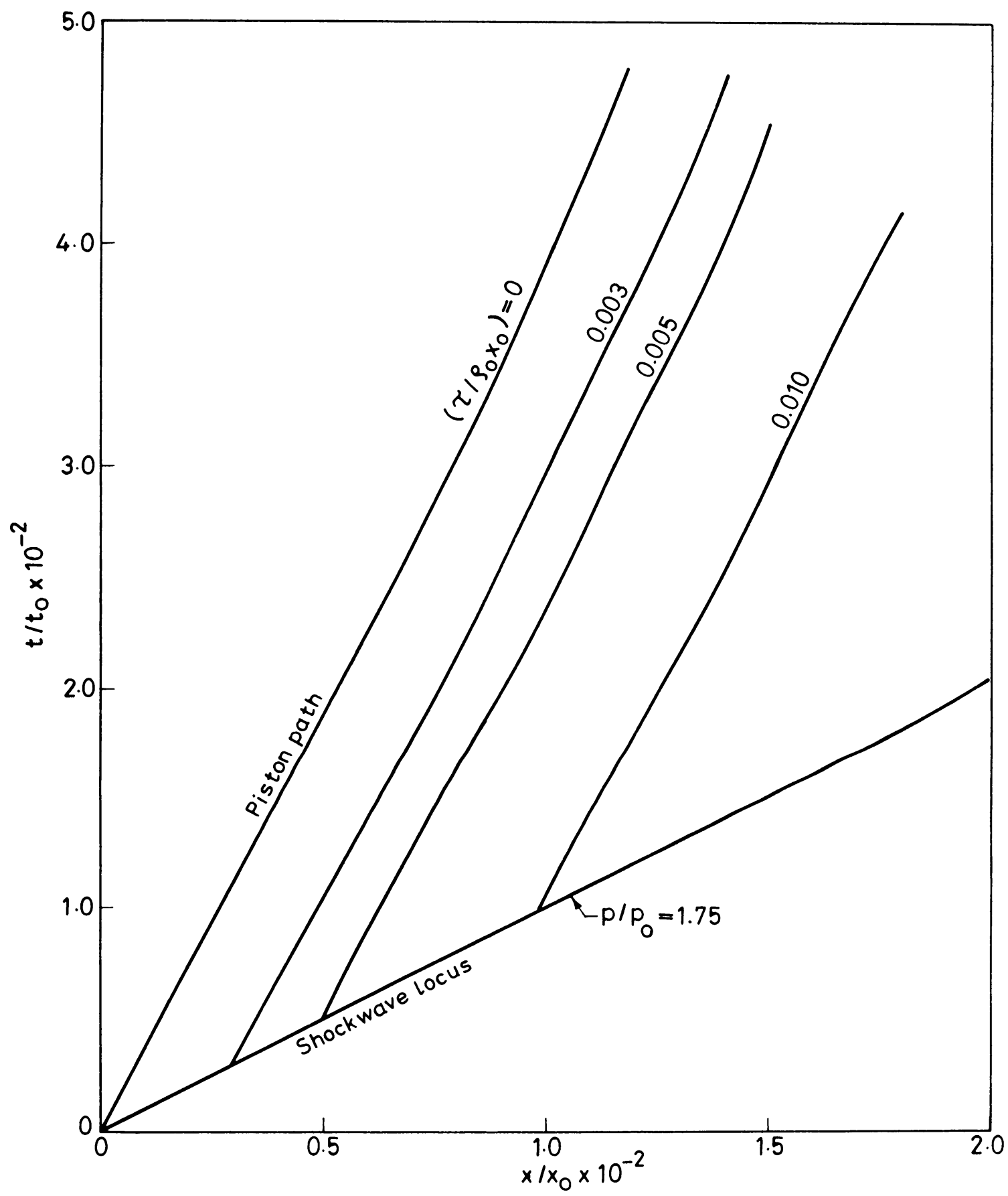

FIG. 7. As in Fig. 5 with $\gamma=\frac{5}{3}, b=\frac{8}{3}, \alpha=-1.5, a=1$. The shock velocity decays to zero in a finite time $\left(t=2 t_{0}\right)$ and a finite distance $\left(x=x_{0} / 2\right)$ in an increasing density medium. The density becomes infinite at $x=x_{0} / 2$. 


$$
a_{4}=\frac{1}{12\left(P_{*}-1\right)}\left[6 a_{3}\left(a_{1}-1\right)+2 a_{2}^{2}\left(2 a_{1}-1\right)-\frac{(\gamma+1)(2 \gamma+1) a_{1} C_{1}}{2 \gamma^{2} P_{*}^{2}}\right] .
$$

Using (4.29), Eqs. (4.24) can be integrated in the neighborhood of $P=P_{*}$ to give the piston path

$$
\begin{aligned}
\frac{x}{x_{0}}= & \frac{1}{a \alpha} \frac{1}{a_{1} b}\left[2\left(P_{*}-1\right) a_{2}\left(P-P_{*}\right)+\left[3\left(P_{*}-1\right) a_{3}+b_{2}\right]\left(P-P_{*}\right)^{2}\right. \\
& \left.+\left[4\left(P_{*}-1\right) a_{4}+2 a_{3}+\frac{2}{3} a_{2}^{2}\right]\left(P-P_{*}\right)^{3}\right]+O\left(P-P_{*}\right)^{4}, \\
\frac{t}{t_{0}}= & \frac{1}{a \alpha} \frac{1}{a_{1}}\left[2 a_{2}\left(P-P_{*}\right)+3 a_{3}\left(P-P_{*}\right)^{2}+4 a_{4}\left(P-P_{*}\right)^{3}\right]+O\left(P-P_{*}\right)^{4} .
\end{aligned}
$$

From Eqs. (4.30) it is clear that, for a physically meaningful solution, that is for $x$ and $t$ positive, we have $P \geq P_{*}$ for $a \alpha>0$, and $1<P \leq P_{*}$ for $a \alpha<0$.

5. Exponential-type similarity solutions. The second class of similarity form of solutions identified by (3.24) imply

$$
\begin{aligned}
w & =\phi W(\sigma), \\
p & =p_{0} P(\sigma), \\
\sigma & =\frac{s}{p_{0} t_{0}} \exp \left(-\frac{a \tau}{\rho_{0} x_{0}}\right), \\
f & =\frac{\gamma p_{0}^{1 / \gamma}}{b \rho_{0}} C_{1} \exp \left(-\frac{a \tau}{\rho_{0} x_{0}}\right), \\
\phi & =\frac{p_{0} t_{0}}{\rho_{0} x_{0}} \exp \left(\frac{a \tau}{\rho_{0} x_{0}}\right) .
\end{aligned}
$$

The constants $x_{0}, t_{0}, p_{0}, \rho_{0}, C_{1}$ and a occurring above are similar to those defined in Sec. 4 . Substitution of (5.1)-(5.5) into (2.17) $-(2.18)$ yields

$$
\begin{aligned}
(W-a \sigma) W^{\prime}-C_{1} P^{-1 / \gamma} P^{\prime} & =a(1-W), \\
P W^{\prime}-(W-a \sigma) P^{\prime} & =0 .
\end{aligned}
$$

We have chosen the undisturbed density to be

$$
\rho_{*}=\rho_{0} \exp \left(-\frac{2 a \tau}{\rho x_{0}}\right)
$$

so as to get the similarity form of the shock conditions. Eqs. (5.6) and (5.7) can be rewritten as

$$
\begin{aligned}
d W / d P & =(W-V) / P, \\
d V / d P & =\left(C_{1} P^{\gamma-1 / \gamma}-(W-V)^{2}\right) / P(W-1),
\end{aligned}
$$

with initial conditions

$$
W\left(P_{*}\right)=P_{*}, \quad V\left(P_{*}\right)=0 .
$$


where $V=a \sigma$. It is instructive to note that Eqs. (5.9) and (5.10) can be recovered from Eqs. (4.10) and (4.11) in the limit of $\alpha$ tending to $\pm \infty$, implying $a_{1}=1$. The solutions without shocks do not exist in the present family.

Proceeding as in Sec. 4, it is easy to verify that the parametric representation of shock wave motion is

$$
\frac{x}{x_{0}}=\frac{1}{2 a}\left[\exp \left(\frac{2 a \tau}{\rho_{0} x_{0}}\right)-1\right] \quad \frac{t}{t_{0}}=\frac{1}{a}\left[\exp \left(\frac{a \tau}{\rho_{0} x_{0}}\right)-1\right] .
$$

The explicit trajectory of the shock, shock velocity, and the undisturbed density ahead of the shock are given respectively by

$$
\begin{aligned}
\frac{x(t)}{x_{0}} & =\frac{1}{2 a}\left(\left(1+\frac{a t}{t_{0}}\right)^{2}-1\right), \\
U(t) & =\frac{x_{0}}{t_{0}}\left(1+a t / t_{0}\right), \\
\rho_{*}(x) & =\rho_{0} /\left(1+2 a x / x_{0}\right) .
\end{aligned}
$$

The functions $x$ and $t$ are found to be

$$
\begin{aligned}
\frac{x}{x_{0}} & =\frac{\exp \left(\frac{2 a \tau}{\rho_{0} x_{0}}\right)}{a b} \int_{P_{*}}^{P}\left[\left(\frac{d W}{d P}\right)^{2}-C_{1} P^{-(\gamma+1 / \gamma)}\right] d P+\frac{1}{2 a}\left[\exp \left(\frac{2 a \tau}{\rho_{0} x_{0}}\right)-1\right], \\
\frac{t}{t_{0}} & \left.=\frac{\exp \left(\frac{a \tau}{\rho_{0} x_{0}}\right)}{a} \int_{P_{*}}^{P}\left[\left(\frac{d W}{d P}\right)^{2}-C_{1} P^{-(\gamma+1 / \gamma)}\right] \frac{d P}{(W-1)}+\frac{1}{a} \exp \left(\frac{a \tau}{\rho_{0} x_{0}}\right)-1\right],
\end{aligned}
$$

where

$$
P_{*} \leq P<\infty, \text { for } a=1 \text { and } 1<P \leq P_{*} \text { for } a=-1 \text {. }
$$

The piston motion corresponding to $\tau=0$ is given by

$$
\begin{aligned}
& \frac{x}{x_{0}}=\frac{1}{a b} \int_{P_{*}}^{P}\left[\left(\frac{d W}{d P}\right)^{2}-C_{1} P^{-(\gamma+1 / \gamma)}\right] d P, \\
& \frac{t}{t_{0}}=\frac{1}{a} \int_{P_{*}}^{P}\left[\left(\frac{d W}{d P}\right)^{2}-C_{1} P^{-(\gamma+1) / \gamma}\right] \frac{d P}{(W-1)} .
\end{aligned}
$$

Some typical results for the presemt class of solutions are shown in Figs. 8 and 9.

The local solution of (5.9) and (5.10) and the piston path in the neighborhood of $P=P_{*}>1$ are given respectively by Eqs. (4.29) and (4.30) with $a_{1}=1$ and $\alpha=1$.

6. Discussion and conclusions. We have in this paper found two families of similarity solutions of a transformed system of one-dimensional gasdynamic equations. The similarity form of the transformed system implies the following constraints: (1) the density in the undisturbed medium (with a constant pressure) varies according to the power law (4.17), which implies either a decreease in density ahead of the shock to zero or to an increase in 


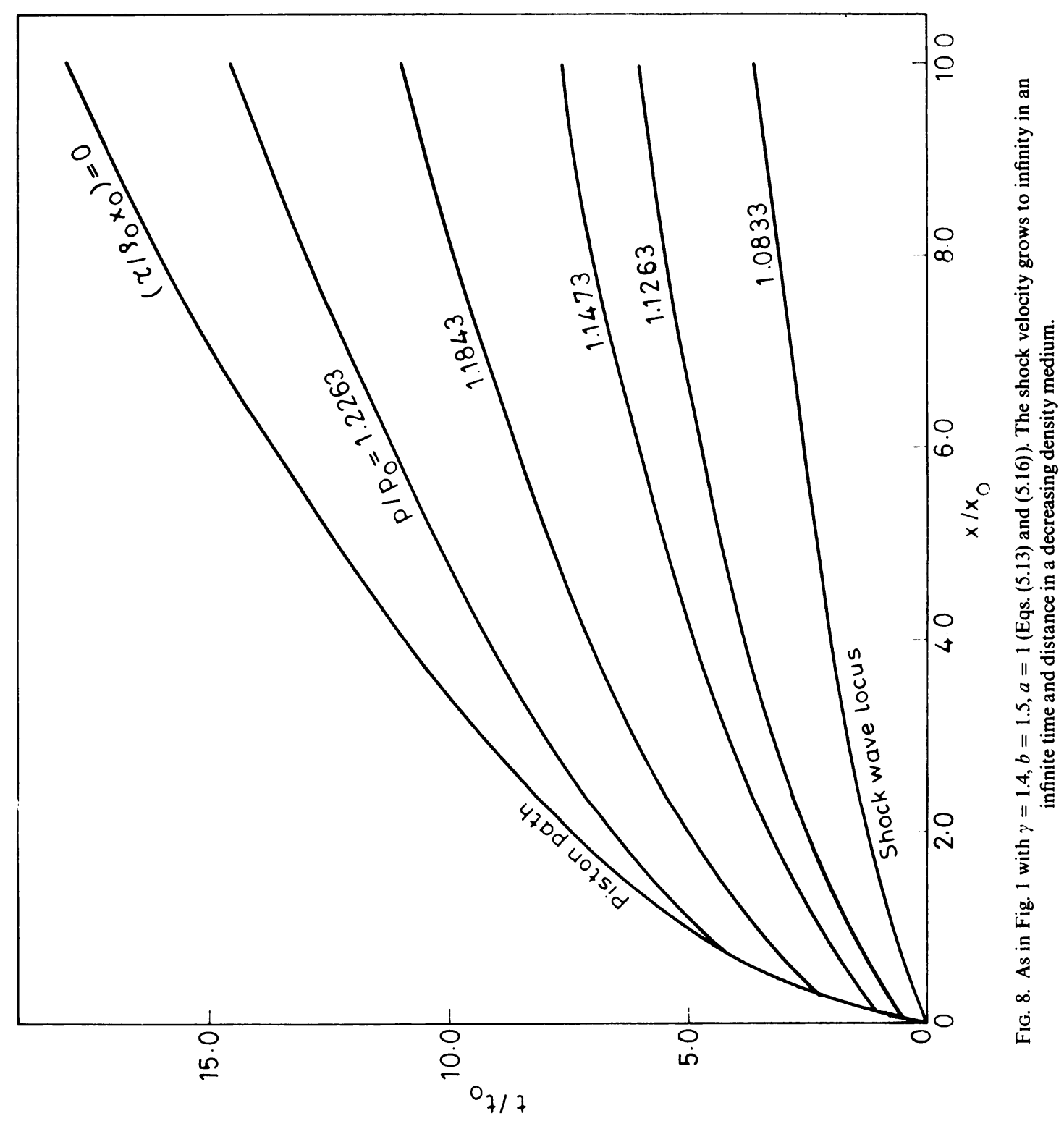




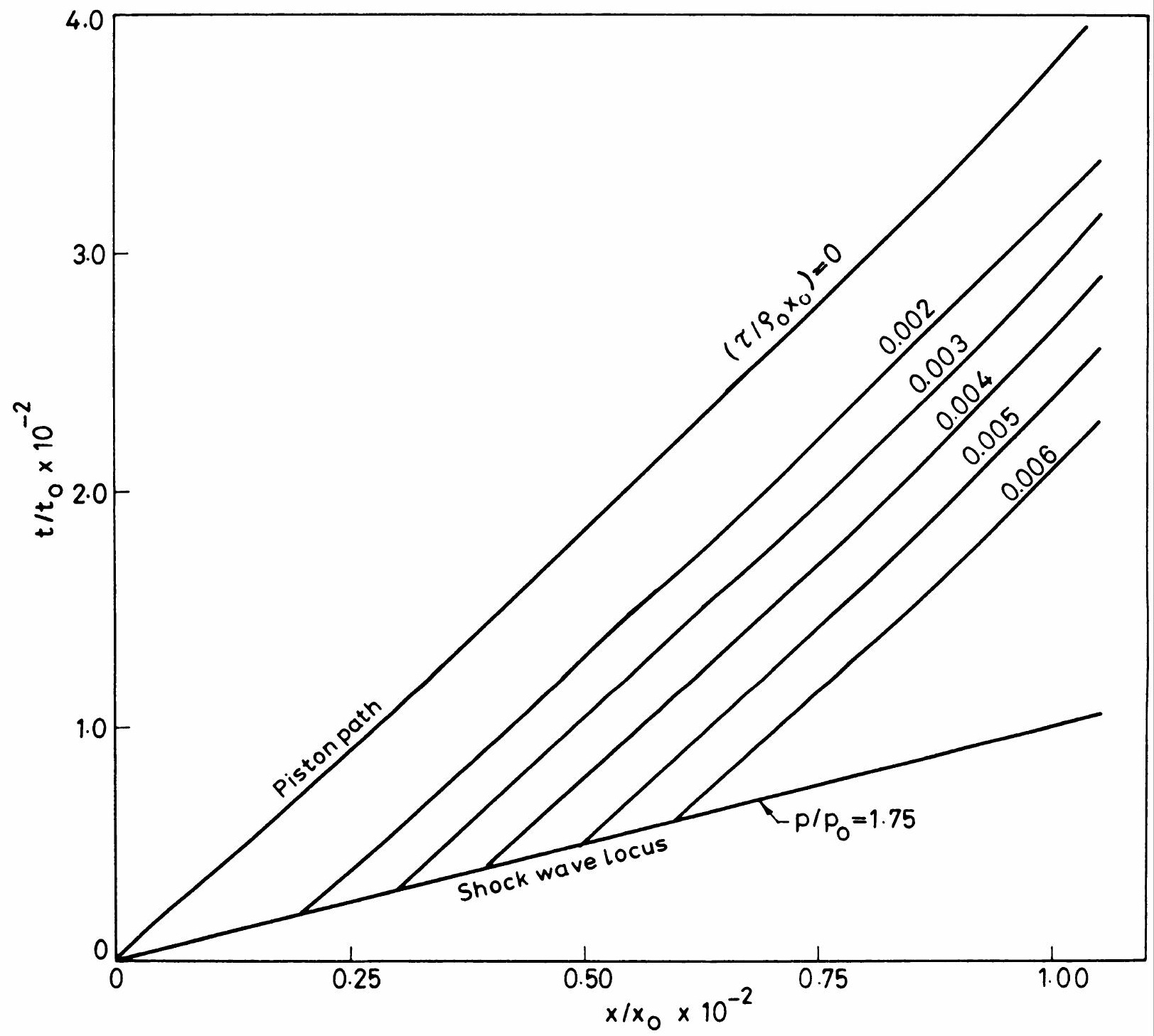

Fig. 9. As in Fig. 5 with $\gamma=\frac{5}{3}, b=\frac{8}{3}, a=-1$. The shock velocity decays to zero in a finite time $\left(t=t_{0}\right)$ and a finite distance $\left(x=x_{0} / 2\right)$ in an increasing density medium. The density becomes infinite at $x=x_{0} / 2$. 
density to an infinite value, at an infinite or a finite distance, (2) the shock strength as measured by pressure ratio across the shock or shock Mach number has a constant value throughout the course of shock propagation, while the shock velocity itself either grows to infinity in the case of a decreasing-density medium or decays to zero in the increasingdensity medium at an infinite or a finite time according to Eq. (4.18).

The propagation of shocks of constant strength has been considered earlier by several authors-for example by Carrus, Haas and Kopal [10], who analyzed the propagation of shocks of constant strength in a compressible spherical gas-configuration which is stratified under the effect of gravity. The shock was assumed to be caused by a central explosion. As Figs. 1-9 show, the piston accelerates (decelerates) in the case of a growing (decaying) shock velocity, moving into a decreasing (increasing) density medium, thus assisting in the process of shock velocity growth (decay). The circumstance of the shock velocity decaying (growing to become infinite) at a finite distance and a finite time is a little unusual, although the increasing (decreasing) density in the undisturbed medium causing it is quite conceivable in spite of the piston motion from behind. In fact, in these cases, the piston stops much before the point where the density becomes zero or infinite and Figs. 6, 7, and 9 show the piston motion up to its final stage. In these cases, although the shock velocity and the sound speed both vanish (or become infinite) at a finite distance and a finite time, their ratio remains constant and equal to its initial value. The decay of an initial profile headed by a shock which evolves according to the single non-linear PDE

$$
\begin{gathered}
u_{t}+g(u) u_{x}+\gamma u^{\alpha}=0, \quad 0<\alpha<1, \quad \lambda>0, \\
g(u)>0, \quad g_{u}(u)>0, \quad 0<u \ll 1,
\end{gathered}
$$

has been considered by Murray [11]. He finds that the profile decays in a finite time and a finite distance. Thus, the case of the medium with increasing density is somewhat similar, though here we consider a boundary-value problem and the damping is affected by the nonuniform undisturbed medium. The propagation of an initially spherically shock wave due to explosion in air in the direction of most rapidly increasing density (besides other directions) has been studied by Zeldovich and Raizer [12], in the limit of the shock having traversed a large distance from the point of explosion. They have brought out clearly the attentuating effect of the increasing density on the shock velocity. They have analyzed the analogy of such a motion with the motion of an inhomogeneous atmosphere set in by impulsive loading, that is, a forward piston motion of a very short duration. A subset of the solutions found in the present paper exhibits a rather extreme situation such that the attenuation (growth) of the shock velocity, caused by a certain piston motion and propagating into an increasing (decreasing) density medium, takes place in a finite distance and a finite time.

The preceding discussion pertains to power-law similarity solutions. The exponential form of similarity solutions shows that the shock velocity either decays to zero in the increasing density medium at a finite distance and finite time or becomes infinite in the case of a decreasing density medium at an infinite distance and infinite time (cf. Eqs. (5.14)(5.15)).

For the sake of comparison, we discuss Ustinov's solution, which comes out as a special case of the power-law solutions of Sec. 4 with $\alpha=-1, a=-1$. Ustinov obtained this solution by considering the product form of solution of the system $(2.17)-(2.18)$ and specialized it to the case when $p$ is a function of $s$ only and $u$ is a product of $\phi(\tau)$ (cf. Sec. 2) and a 
function of $s$. He was able to find an explicit solution which shows that the shock velocity grows exponentially with time as it proceeds into a medium of decreasing density. On the other hand, if we take $a=+1$ in (4.25), it can be easily verified that the solution has a shock velocity decaying exponentially at a finite distance $x=x_{0}$, where the undisturbed density blows up. As opposed to Ustinov's solution, our special explicit solution with $\alpha=-\frac{1}{2}$, $\mathrm{a}=1$, shows that the undisturbed density increases exponentially and the shock velocity decays algebraically in time. The case $\alpha=-\frac{1}{2}, a=-1$ gives the solution with a growing shock velocity in a medium with exponentially decreasing density and the shock velocity becomes infinite in a finite time, $t=2 t_{0}$.

Finally, we briefly comment on the solutions which are headed by sonic lines (characteristics) and not shocks. While the solutions with shocks for the power law case exist for $-\infty<\alpha<\infty, \alpha \neq 0$, the shockless ones exist only in the range $-2<\alpha<0$ of the similarity parameter. The case $\alpha=0$, as we have remarked earlier, is inadmissible since it invalidates the basic assumption of non-isentropy in the transformation of the usual system of gasdynamic equations. The exponential form of the similarity variable allows no solutions which are shockless.

\section{REFERENCES}

[1] L. I. Sedov, Similarity and dimensional methods in mechanics, Academic Press, New York, 1959

[2] J. B. Keller, Spherical, cylindrical and one-dimensional gas flows, Quart. Appl. Math. 14, 2, 171-184 (1956)

[3] G. C. McVittie, Spherically symmetric solutions of the equations of gas dynamics, Proc. Roy. Soc. (London) 220, 339-355 (1953)

[4] M. D. Ustinov, Ideal gas flow behind a finite-amplitude shock wave, Izv. AN SSSR Mekh. Zhid. I Gaza (Fluid dynamics) 2, 1, 88-90 (1967)

[5] S. P. Castell and C. Rogers, Applications of invariant transformations in one-dimensional non-steady gas dynamics, Quart. Appl. Math. 32, 241-251 (1974)

[6] M. D. Ustinov, Transformation and some solutions of the equations of motion of an ideal gas, Izv. AN SSSR Mekh. Zhid, I Gaza 3, 68-74 (1966)

[7] G. W. Bluman and J. D. Cole, Similarity methods for differential equations, Springer-Verlag, New York, 1974

[8] L. V. Ovsjannikov, Group properties of differential equations, translation by G. W. Bluman of Gruppovye Svoysta Differentsialny Uravneni, Novosibirsk, U.S.S.R., 1962

[9] J. D. Logan and J. D. J. Perez, Similarity solutions for reactive shock hydrodynamics, SIAM J. Appl. Math. 39, 512-527 (1980)

[10] Pierre A. Carrus, Phyllis A. Fox, Feliz Haas, and Zdenek Kopal, The propagation of shock waves in a stellar model with continuous density distribution, AP. J. 113, 496-518(1951)

[11] J. D. Murray, Perturbation effects on the decay of discontinuous solutions of nonlinear first order wave equations, SIAM J. Appl. Math. 19, 2, 135-160(1970)

[12] Ya. B. Zel'dovich and Yu. P. Raizer, Physics of shock waves and high-temperature hydrodynamic phenomena, Academic Press, New York and London, 1967 